\title{
Effect of the Reference Line on Main Roughness Parameters
}

\author{
R. Fernández ${ }^{1, a}, A . S a n z^{1, b}, S$. Pindado ${ }^{2, c}$ and P. Núñez ${ }^{3, d}$ \\ ${ }^{1,2}$ Department of Aerospace Materials and Production. ETSI Aeronáuticos-UPM. \\ Plaza Cardenal Cisneros 3, 28040 Madrid, Spain \\ ${ }^{2}$ University Institute of Microgravity "Ignacio Da Riva". IDR/UPM. ETSI Aeronáuticos-UPM. \\ Plaza Cardenal Cisneros 3, 28040 Madrid, Spain \\ ${ }^{3}$ ETSI Industriales-UCLM, Avda. Camilo José Cela, 3, 13071- Ciudad Real, Spain \\ aroberto.ffernadez@alumnos.upm.es, ba.slobera@upm.es, csantiago.pindado@upm.es, \\ dpedro.nunez@uclm.es
}

Keywords: microgeometry, surface finishing, roughness, waviness, reference lines

\begin{abstract}
In the present paper the influence of the reference system with regard to the characterization of the surface finishing is analyzed. The effect of the reference system's choice on the most representative surface finishing parameters (e.g. roughness average $R a$ and root mean square values $R q$ ) is studied. The study can also be applied to their equivalent parameters in waviness and primary profiles. Based on ISO and ASME standards, three different types of regression lines (center, mean and orthogonal) are theoretically and experimentally analyzed, identifying the validity and applicability fields of each one depending on profile's geometry.
\end{abstract}

\section{Introduction}

Surface finishing, considered as the study of microgeometrical properties of the workpieces' surfaces and their influence on their functional behaviour, has a great importance in the engineering of the manufacturing processes due to the wide variety of functional behaviours affected by it.

Without underrating other applications, the most widespread techniques in the measurement and characterization of the degree of surface finishing, are based on the definition of a profile using the information provided by a roughmeter with an inductive feeler of contact. The delimitation of the measurement area and the choice of a suitable reference system are key factors in the determination of the values of the most representative parameters used in the characterization of surface finishing. These parameters allow the comparison of measurements done on different zones of the surface or using different measuring instruments which ensure the repeatability of measurements.

Technological importance linked to the study and characterization of surface finishing becomes evident due to the consideration that this topic receives in most bibliography related to manufacturing processes.

In some cases surface finishing is tackled in a monographic way [1], while in other studies is analyzed in some chapters [2]. Also, Spanish and international rules reflect the importance of the study of surface finishing. Among the wide variety of standards, it is remarkable those ones made by ISO [3] and ASME [4], which are the base of most national standards. These rules have experienced many reviews throughout last years. This fact reveals the importance of the subject and the constant search of improvements in the definition, measurement and characterization of surface finishing.

Many parameters, almost all numerical, are used to characterize surface finishing. Among them, average roughness $R a$ and RMS roughness $R q$ are the most widespread. All the parameters require the definition of the region where they are evaluated as well as the filtering characteristics of the information used in its determination, because otherwise, the results are not comparable. This paper is focused on the definition of the reference system for which main roughness parameters, such as $R a$ and $R q$, are obtained. 


\section{Reference system}

In a simplified way, it is possible to say that the mechanical roughmeter receives from the scanning unit a signal proportional to the relative displacements (z-axis) of the stylus' tip respect the trajectory that the measuring head follows in its displacement ( $x$-axis). This signal is processed and filtered internally and is converted in a sequence of digital values $\left(x_{i}, z_{i}\right)$ which correspond to the primary $(P)$, roughness $(R)$ and waviness $(W)$ profiles, from which the parameters that characterize surface finishing are determined. Roughmeters do the required operations internally in order to obtain the parameters, without any possible user's interaction in this calculation process. In fact, if the numerical information of the values which constitute the profile is not available, it is only possible to accept the results and therefore the calculation method used. As it is shown later, the choice of the reference system can cause significant variations in the results, depending on the profile's shape. Determination of the reference system requires the definition of $z$ and $x$-axis direction, the definition of the origin and the definition of the interval which is going to be studied. Present study is focused on the position of $z$-axis, $x$-axis and the origin, assuming that the length in which parameters' values are determined is the required length according to the rules (basic length or evaluation length).

When the digitized information $\left(x_{i}, z_{i}\right)$ corresponding to any of the profiles is available, both ISO and ASME standards define $x$-axis from the concept of least squares line. In general, this line is a straight line obtained by imposing the least squares condition to the distances between profiles' points and the line.

The term "least squares line" presents some ambiguity due to the existence of many possibilities. In this case, the three lines shown in Table 1 are going to be considered.

Table 1. Least squares lines

\begin{tabular}{cccc}
\hline Common name & Minimizes & $\begin{array}{c}\text { Analytical } \\
\text { Expression }\end{array}$ & Acronym \\
\hline Center line & Sum of the squares of vertical distances & $z=b$ & $C L$ \\
Mean line & Sum of the squares of vertical distances & $z=m \cdot x+b$ & $M L$ \\
Orthogonal line & Sum of the squares of orthogonal distances & $z=m \cdot x+b$ & $O L$ \\
\hline
\end{tabular}

Center line is the horizontal least squares line. Its determination is the easiest one because its slope is null and the distances from profile's points to it matches with the ordinates $z_{i}$ of the points. Coefficient $b$ is determined with Eq. 1 .

$$
b=\frac{1}{n} \sum_{i=1}^{n} z_{i}
$$

In Eq. 1, $n$ represents the number of points of the digitized profile.

Mean line minimizes the sum of the squares of vertical distances from the points to it. Unlike the center line, mean line doesn't need to be parallel to abscissa axis. In this case, coefficients $m$ and $b$ are obtained from Eq. 2.

$$
m=\frac{n \sum_{i=1}^{n} x_{i} z_{i}-\left(\sum_{i=1}^{n} x_{i}\right)\left(\sum_{i=1}^{n} z_{i}\right)}{n \sum_{i=1}^{n} x_{i}^{2}-\left(\sum_{i=1}^{n} x_{i}\right)^{2}} ; \quad b=\frac{1}{n} \sum_{i=1}^{n} z_{i}-\frac{m}{n} \sum_{i=1}^{n} x_{i}
$$

Finally, orthogonal line is the least squares line which minimizes the sum of the squares of the distances from the points to it. It has the same analytical expression as the mean line, but coefficients $m$ and $b$ are calculated using Eq. 3 and Eq. 4 . 


$$
\begin{aligned}
& m=\frac{-u \pm \sqrt{u^{2}+4 v^{2}}}{2 v} ; \quad b=\frac{1}{n} \sum_{i=1}^{n} z_{i}-\frac{m}{n} \sum_{i=1}^{n} x_{i} \\
& u=\frac{1}{n}\left(\sum_{i=1}^{n} x_{i}^{2}-\sum_{i=1}^{n} z_{i}^{2}\right)-\frac{1}{n^{2}}\left(\left(\sum_{i=1}^{n} x_{i}\right)^{2}-\left(\sum_{i=1}^{n} z_{i}\right)^{2}\right) ; v=\frac{1}{n} \sum_{i=1}^{n} x_{i} z_{i}-\frac{1}{n^{2}}\left(\sum_{i=1}^{n} x_{i}\right)\left(\sum_{i=1}^{n} z_{i}\right)
\end{aligned}
$$

In order to highlight the importance of the choice of the line in the value of the parameters, let's suppose a profile consisting of isosceles triangles as shown in Fig.1. This profile is only used as a supporting element of the exposition, but it does not represent a real situation because the values of its heights are greater than those ones which can appear in a workpiece.

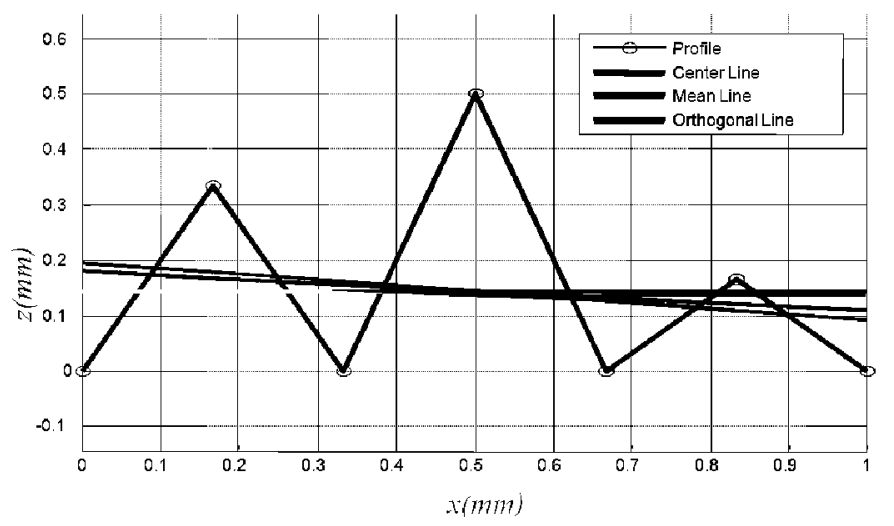

Fig. 1. Sample profile

The determination of the values of $R a$ and $R q$ depending on the line conducts to the results shown in Table 2.

Table 2. Values for the example profile (Fig. 1)

\begin{tabular}{cccc}
\hline Line & Equation & $\boldsymbol{R a}$ & $\boldsymbol{R q}$ \\
\hline $\boldsymbol{C} \boldsymbol{L}$ & $z=0.14286$ & 0.1633 & 0.1875 \\
$\boldsymbol{O} \boldsymbol{L}$ & $z=-0.10336 x+0.19454$ & 0.1624 & 0.1853 \\
$\boldsymbol{M L}$ & $z=-0.071429 x+0.17857$ & 0.1629 & 0.1855 \\
\hline
\end{tabular}

It is possible to see that the values of the parameters referred to $M L$ and $O L$ lines hardly present variation, while the values referred to $C L$ present a greater variation. Also, the value associated to $O L$ is smaller than $M L$ and $C L$ values. Although it is a particular case, this fact suggests that, for the same measurement according to ISO and ASME standards, the choice of $O L$ as reference line conducts to smaller values of the parameters $R a$ and $R q$.

\section{Methodology}

To determine the order of magnitude of the difference between the values of the calculated parameters referred to different lines it is important to consider the relationship between the maximum height of the profile and its length. In fact, if the maximum height of the profile is designated as $H$ and the length in which the parameters are evaluated as $L$, the greater the ratio $H / L$ is, the greater is the difference due to the choice of the reference line. For small values of $H / L$, that difference decreases even becoming negligible.

a. Influence of the parameter $H / L$ on the slope of least squares lines

Using a profile with the same form as the example profile shown in Fig. 1, it can be observed that if the parameter $H / L$ is modified, the slope of the orthogonal and mean line experienced a variation as shown in Fig. 2. It is observed that when the $x$-coordinate is dominant over $z$ (small values of $H / L$ ) the slope of both lines tends to zero, while when $z$-coordinate is dominant (great values of $H / L$ ) the slope in absolute value increases considerably. 


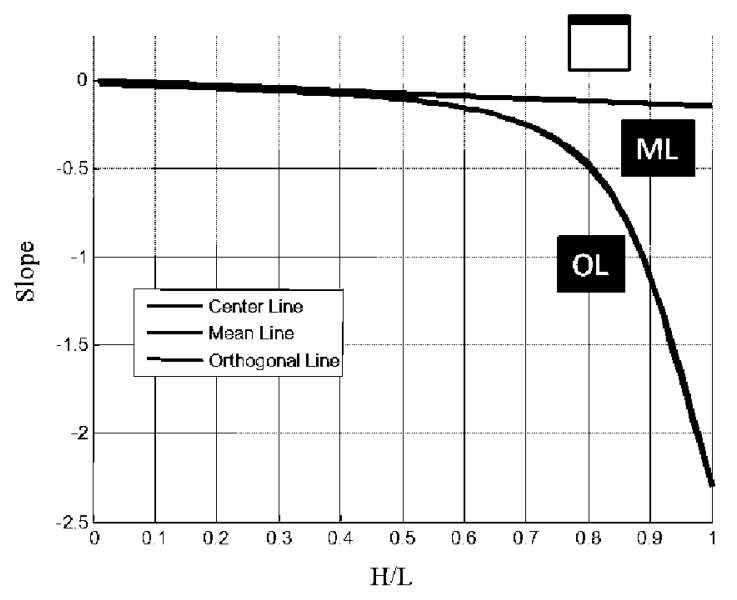

Fig. 2. Slope vs. ratio $\mathrm{H} / \mathrm{L}$ in the reference lines

\section{b. Mathematical basis}

Although previous results have been obtained working on a particular profile, the behaviour of the slope of ML and OL versus the ratio $H / L$ is qualitatively similar. Analytical expressions, whose minimization conducts to the values of the lines' coefficients, are shown in Eq. 5 and Eq. 6 for the mean line and orthogonal line, respectively.

$$
\begin{aligned}
& f(m, b)=\sum_{i=1}^{n}\left(m \cdot x_{i}+b-z_{i}\right)^{2} \\
& f(m, b)=\sum_{i=1}^{m} \frac{1}{m^{2}+1}\left(m-x_{i}+b-z_{i}\right)^{2}
\end{aligned}
$$

If profile's length is kept constant, $H / L$ decreases due to the reduction of the z-coordinate and therefore the slope of both lines. In expressions (5) and (6), it is possible to check when $m$ tends to zero that $m^{2}$ is much smaller than $m$, which is much smaller than 1 . As a result, both expressions converge to the same function.

Table 3. Normalized values of sampling length and $\mathrm{Ra}$

\begin{tabular}{lllllllllll}
\hline Sampling length $(\mathbf{m m})$ & & & 0.08 & 0.25 & 0.8 & 2.5 & 8 & & \\
\hline $\boldsymbol{R} \boldsymbol{a}(\boldsymbol{\mu m})$ & 0.1 & 0.2 & 0.4 & 0.8 & 1.6 & 3.2 & 6.2 & 12.5 & 25 & 50 \\
\hline
\end{tabular}

In order to have an approximation to the order of magnitude of $\mathrm{H} / \mathrm{L}$, the normalized values of basic lengths and average roughness $R a$ (Table 3 ) are considered. It can be observed that H/L hardly overcomes the value 2.5 being its most usual range of variation the interval $[0.0005 ; 2.5]$.

\section{Results}

If we represent the value of the parameters of roughness calculated for the example profile proposed in the previous paragraph, we obtain the results shown in Fig. 3. The value of the parameters $R a$ and $R q$ calculated respect the three reference lines is represented. In ordinates, it has been represented the difference between each parameter calculated respect each couple of lines. It has been used as nondimensionalization element the value of the parameter calculated respect to CL, i.e., they appear in the form

$$
\frac{R_{L 1}-R_{L 2}}{R_{L E}}
$$


where L1 and L2 represent a couple of different lines and $R$ represents parameters $R a$ and $R q$ depending on the graph. It has been chosen an interval of variation of $H / L$ from 0 to 0.2 , which involves most real situations of measurement.
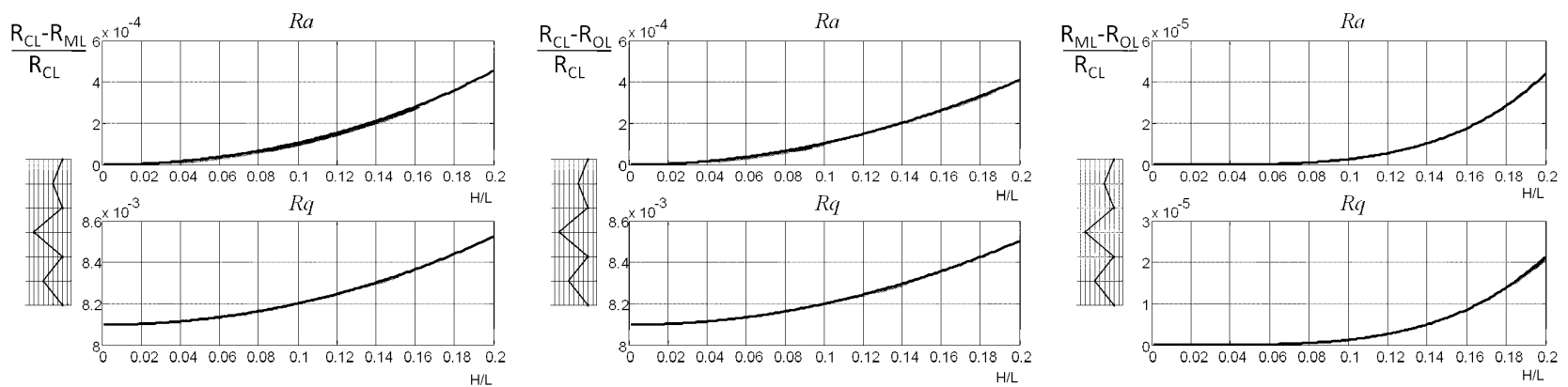

Fig. 3. Variation of roughness parameters due to $\mathrm{H} / \mathrm{L}$ (sample profile)

It can be checked that the difference between the parameters decreases with $H / L$ independently the line which is considered. Likewise it can be observed as the graphs which relate the calculated parameters in the lines CL-ML and CL-OL are almost equal with values slightly greater in the case of CL-OL. This fact is corroborated in the comparison shown for lines ML-OL where it is noticeable that the order of magnitude is notably lower, about $10^{-5}$ versus $10^{-4}$ and $10^{-3}$ (depending if we are considering $R a$ or $R q$ ) of the comparisons CL-ML and CL-OL.

Following a similar procedure as the one described for the example profile, work has been done with real data obtained from a series of 20 cylindrical workpieces machined in a numerical control turning machine with different values of feed and cutting speed. All the workpieces were measured in a roughmeter with an inductive feeler of contact. Resultant values were quite similar in all the workpieces. For graphical representation it was chosen the data from a workpiece machined with a feed $f=0.3 \mathrm{~mm} / \mathrm{rev}$ (Fig. 4). In order to obtain the roughness profile, measurements have been done along 5 basic lengths of $0.8 \mathrm{~mm}$.

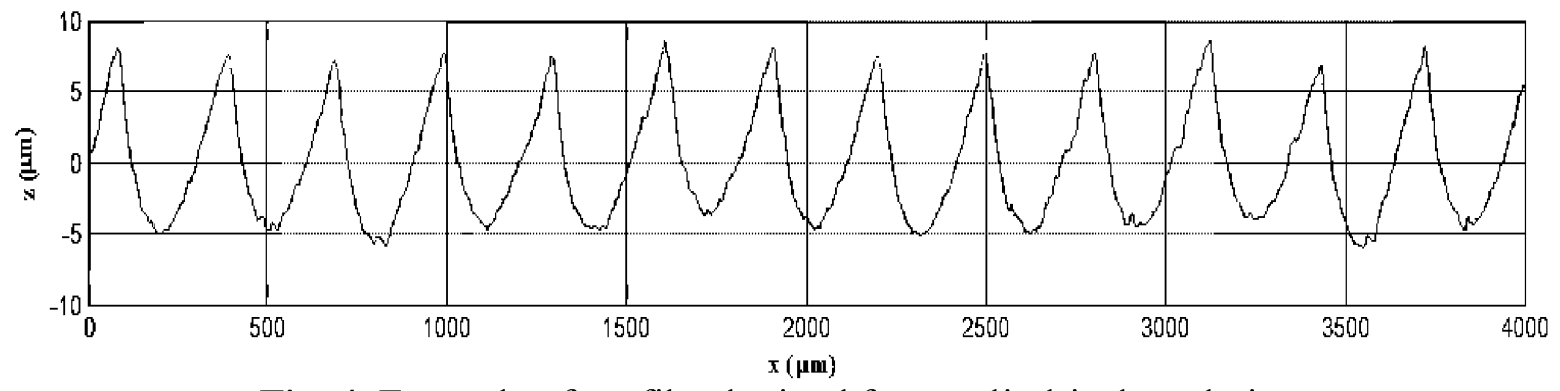

Fig. 4. Example of profile obtained from cylindrical workpiece

Table 4 presents the values of $H / L$ in every basic length. It can be observed very similar values in all the cases.

Table 4. Values $H / L$ in every basic length of the measured profile

\begin{tabular}{cccccc}
\hline & $\boldsymbol{l}_{\boldsymbol{b} 1}$ & $\boldsymbol{l}_{\boldsymbol{b} 2}$ & $\boldsymbol{l}_{\boldsymbol{b} 3}$ & $\boldsymbol{l}_{\boldsymbol{b} 4}$ & $\boldsymbol{l}_{\boldsymbol{b} \mathbf{5}}$ \\
\hline$H / L$ & 0.0100 & 0.0098 & 0.0108 & 0.0108 & 0.0103 \\
\hline
\end{tabular}

Results about real workpieces are collected in Fig. 5. In the representation, it has been followed the same criteria as in Fig. 3. As it was expected, resultant values are considerably smaller than those ones estimated for the example profile, because the ratio $H / L$ is smaller in this case. It is remarkable that the difference between the parameters measured respect ML and OL is about $10^{-7}$. 

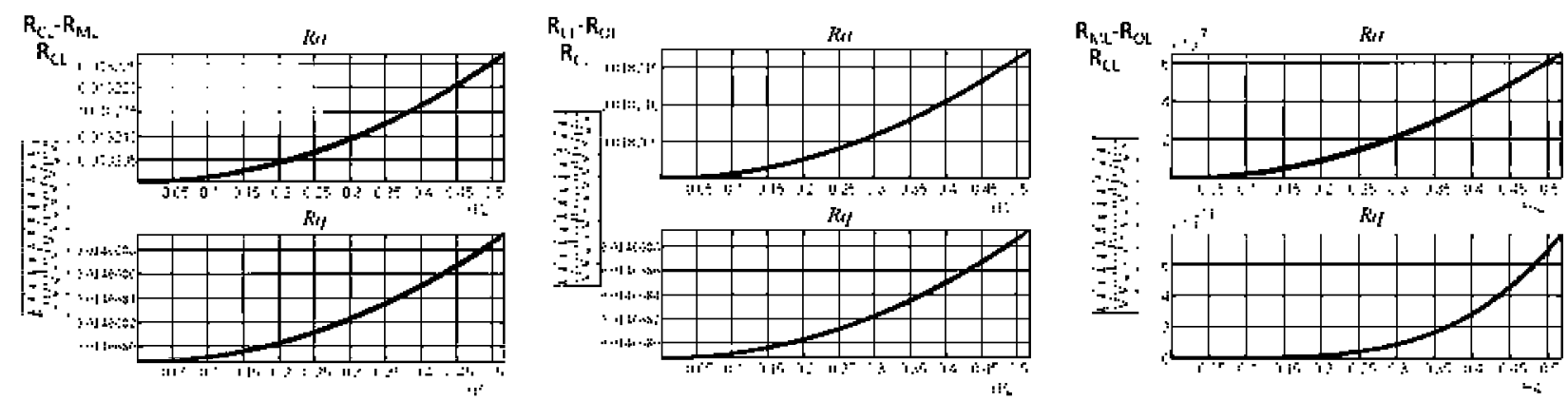

Fig. 5. Variation of roughness parameters due to $\mathrm{H} / \mathrm{L}$ (cylindrical workpiece)

\section{Conclusions}

Surface finishing characterization using numerical parameters require a detailed definition of the reference system for which the calculations are done. Once the reference system has been chosen, it is necessary to determine the situation of the abscissa axis. It is defined by the rules as a least squares line, but there is no clear indication how it is determined. In the present study it has been done a study about the variation experienced by the two principal roughness parameters $R a$ and $R q$, depending on the three types of least squares lines, designated as center line CL, mean line ML and orthogonal line OL. First line, CL, is the easiest one to calculate and it is defined imposing parallelism condition respect the displacement direction of the roughmeter when it is getting data. The second line, ML, is the regression line in ordinates traditionally employed in other fields of engineering in which regression adjustments are required. The third line, OL, is the least used despite being "the real least squares line", because of all the possible lines which satisfy that condition is the only one in which the sum of the squares of the distances from the points of the profile to it is minimum. Among the three lines, OL generates the smaller values using the same initial data. According to the results, the main conclusion is that the use of ML and OL conducts to the same values for $R a$ and $R q$ because the difference between them is smaller than the usual resolution of the roughmeters, which is about $0.01 \mu \mathrm{m}$. Another conclusion is that for most values of the ratio $H / L$ (profile's height/ profile's length), the use of the center line is acceptable. In any case, if it is possible, it is convenient to have the data of the profile's points instead of trusting, without another check, in the values supplied by the roughmeters.

\section{References}

[1] T. R. Thomas: Rough Surfaces (Imperial College Press, 2nd Edition, 1999).

[2] S. Kalpakjian and S. Schmid: Manufacturing Engineering and Technology (Prentice Hall, 6th Edition, 2009).

[3] UNE-EN ISO 4287:1999, Especificación geométrica de productos (GPS). Calidad superficial: Método del perfil. Términos, definiciones y parámetros del estado superficial. (ISO 4287:1997+Technical Corrigendum 1).

[4] ASME B46-1-2002. Surface Texture (Surface Roughness, Waviness, and Lay), 2002. 